\title{
The Effectiveness of Concept Mapping as an Educational Nursing Intervention Strategy to Handle Diabetic Foot: Integrative Review
}

\author{
Reem Mohammad Al Johani ${ }^{*}$, Elham Al Nagshabandi² \\ ${ }^{1}$ Nursing Administration Department, Yanbu General Hospital, Yanbu, KSA \\ ${ }^{2}$ Faculty of Nursing, King Abdulaziz University, Jeddah, KSA \\ Email: ^Reem-ALJohani-88@hotmail.com
}

How to cite this paper: Al Johani, R.M. and Al Nagshabandi, E. (2020) The Effectiveness of Concept Mapping as an Educational Nursing Intervention Strategy to Handle Diabetic Foot: Integrative Review. Open Journal of Nursing, 10, 143-154 https://doi.org/10.4236/ojn.2020.102009

Received: December 29, 2019

Accepted: February 15, 2020

Published: February 18, 2020

Copyright $\odot 2020$ by author(s) and Scientific Research Publishing Inc. This work is licensed under the Creative Commons Attribution-NonCommercial International License (CC BY-NC 4.0).

http://creativecommons.org/licenses/by-nc/4.0/ (c) (i) (5) Open Access

\begin{abstract}
Introduction: Concept map is used as a teaching strategy, where nursing uses this method often to learn more effectively and to be able to apply knowledge when working with diabetic patients. Objective: The aim of this review is to examine all the published articles related to the effectiveness of concept mapping as an educational nursing intervention strategy to handle diabetic foot from 2010-2019. Methodology: Integrative review design in reviewing pertaining literature. Key words related to research were used, database obtained from Cochrane library, MEDLINE, PubMed, Science Direct, Cumulative Index Nursing and Allied Health Literature (CINAHL), ProQuest and Ovid databases were searched, covering the period 2010 to 2019. Other Studies were included if they were related to the effectiveness of concept mapping as an educational nursing intervention strategy to handle diabetic foot. Results: Reveled that concept mapping is a very crucial education tool for both nurses and patients. It is used by nurses to teach diabetic patients how to prevent diabetic foot ulcer, as well as to understand how to organize information and understand the facts. Conclusion: This review confirmed that there are growing confirming literatures about the application of concept maps as an effective nursing strategy. It that can be used by nurses and other health care professionals, to maintain healthy feet and prevent further complications such as bruises, cuts and sores.
\end{abstract}

\section{Keywords}

Concept Map, Diabetic Foot Care, Educational Intervention, Type 2 Diabetic Patients 


\section{Introduction}

Diabetes mellitus is well-defined as a disorder of carbohydrates metabolism categorized by inadequate production of insulin by body thereby creating an improper maintenance level of glucose in the blood sugar [1]. It is a chronic disease which can result from inheritance or acquire a deficiency relating to insulin production by the pancreas or even disturbances in the production of insulin making it inefficient. Having a deficiency in insulin production may end in a high concentration of glucose in the blood and hence damaging many body tissues more so the nerves and blood vessels [1].

Diabetes mellitus has two forms, type 1 diabetes and type 2 diabetes which is caused by different factors, with type 2 diabetes being the most common one in the world. With the two types of diabetes being associated with insulin presence or lack of, type 2 diabetes can also be caused by one having certain genes mutations. Research conducted has shown that millions of people in the world suffer from diabetes such as Saudi Arabia being reported among the top ten countries that have the highest number of patients with diabetes [2]. From researches done on available literature, most people suffering from diabetes in Saudi Arabia are male, those who are living in urban areas suffer more from diabetes than those living in rural areas. Whish confirms that diabetes is spreading across Saudi Arabia fast and more and more people are suffering from diabetes [3].

Concept maps are diagrams that represent the relationships between different variables. Concept mapping in health education is an important part of the management of diabetic foot care. This education tool ensures that nurses are well prepared and equipped when working with patients with diabetes type 2 . This resulted in better patient care and improved the overall efficiency of the nurses [4].

\section{Methods of the Review}

This review is aimed to examine the effectiveness of concept mapping as an educational nursing intervention strategy to handle diabetic foot. It incorporates two-steps search strategies to identify published and unpublished studies. The first step involved an extensive search in different databases which are: Cochrane library, MEDLINE, PubMed, Science Direct, Cumulative Index Nursing and Allied Health Literature (CINAHL), ProQuest and Ovid. The period 2010-2019 was specifically selected to include all potential studies. The choice of this period of time was mainly to detect significant studies published in given years. The second step included a manual or hand-search of the reference lists and bibliographies of all studies highlighted as part of step one.

\subsection{Inclusion and Exclusion Criteria}

The inclusion criteria comprised the studies that address diabetic foot care aspects such as prevention, treatment, management, diabetic foot care, concept 
mapping, self-efficacy behavior, type 2 diabetic, and health education tools., published between 2010-2019 and in English language. While the exclusion criteria for this review included all studies that include foot care among type $1 \mathrm{di}$ abetes patients and articles that were not peer-reviewed.

\subsection{Search Terms}

The search started by dividing the review aims into two themes to facilitate an easier and more importantly a more accurate search process. Thus, the definitive search terms used in this study were:

1) The first theme is self-efficacy behavior prevention and management of diabetic foot care including six sub-themes of: Diabetic Mellitus; type 2 diabetes, diabetic foot, early detection of diabetic foot ulceration and self-efficacy behavior.

2) The Second theme is Concept mapping in health education including three sub-themes of: health education tool, foot care, concept map prevention and management of diabetic foot care. To illustrate how these terms were used, the search commenced with each keyword, and was further developed by grouping and combining terms together by using Boolean operators to broaden or narrow the combined key words or phrases by using AND and OR across all databases.

\subsection{Literature Search Results}

The total number of the identified literature was screened in accordance with the PRISMA (Preferred Reporting Items for Systematic Reviews and Meta-Analysis) four-phase flow diagram. PRISMA explained in Figure 1. The initiative search was conducted founding 2430 hits. The search was narrowed to 1737 articles according to the inclusion and exclusion criteria were established.870 articles were eliminated due to duplicated studies. The abstract was screened also for the relevancy of the search and 123 were excluded. The articles were narrowed to 60 when assessing full-text articles for eligibility. Both published and non-published articles of descriptive longitudinal retrospective study, experimental study, observational study, cohort studies, case study, and literature reviewed study were included in the search.

\subsection{Synthesis of Evidence}

The synthesis involved a wide variety of studies based on different aspects of concept mapping related to diabetic foot. Each study included was reviewed individually by comparing each paper's aims and/or objectives, study design and analysis methods, conclusion of the study findings and implications for practice. This process helped to highlight common themes emerging across the studies and identify what the studies shared in common and what differences could be isolated from them. Figure 1 illustrates the PRISMA flow diagram to show the selection process of the included studies. 


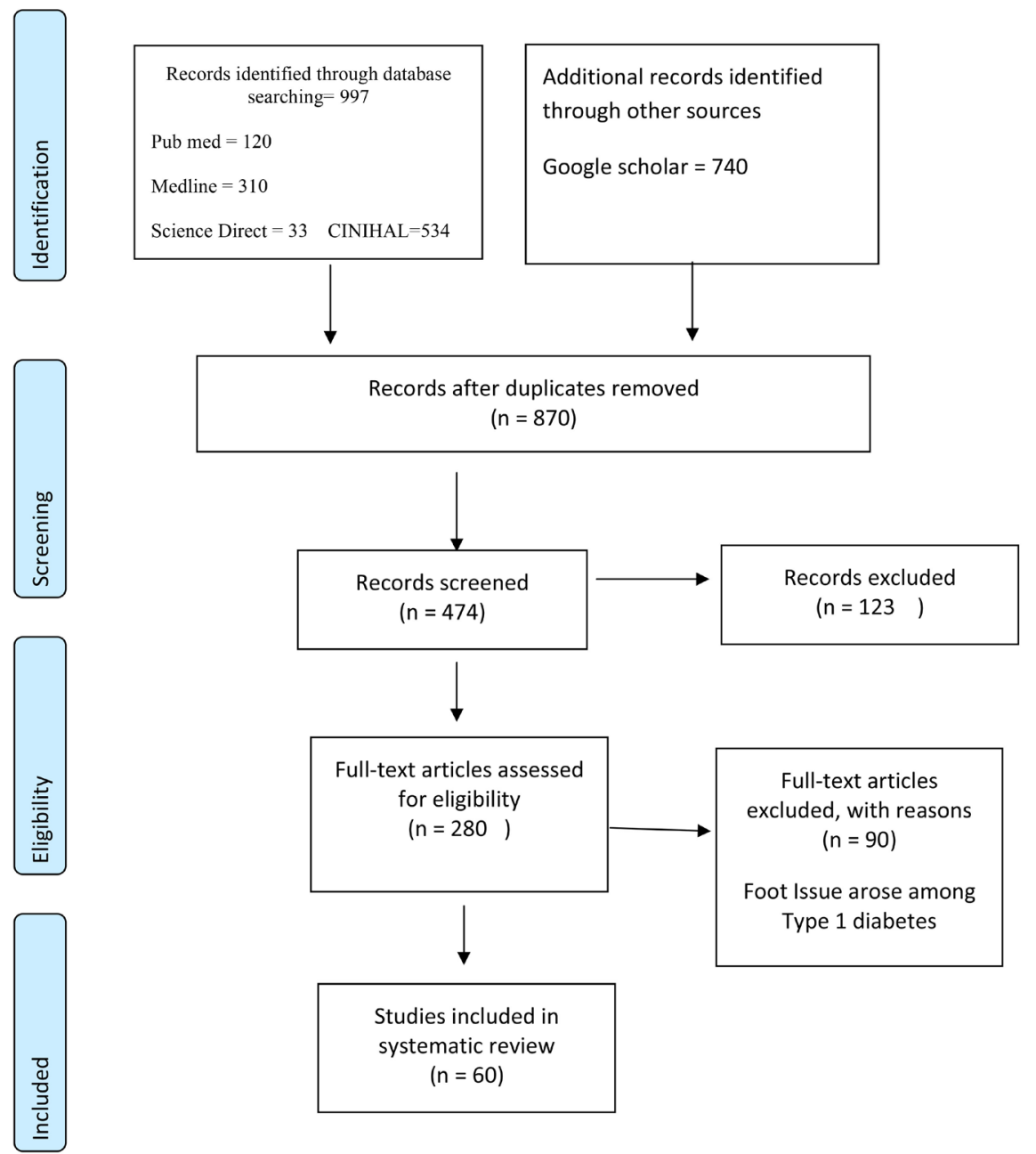

Figure 1. PRISMA of the Study. From: "Moher D, Liberati A, Tetzlaff J, Altman DG, the PRISMA Group (2009)”. Preferred Reporting Items for Systematic Reviews and Meta-Analyses: The PRISMA Statement. PLoS Med 6(7): e1000097. doi:10.1371/journal.pmed1000097.

\subsection{Results}

\subsubsection{Theme 1: Self-Efficacy Behaviour Prevention and Management of Diabetic Foot Care}

\section{1) Sub-Theme 1: Diabetes Mellitus}

Diabetes mellitus is primarily categorized by hyperglycemia owing to imbalances in the secretion of insulin. Hyperglycemia is linked to dysfunction and long-term damage to numerous body organs such as kidneys, blood vessels, eyes, heart, and nerves. Diabetes is currently on the rise worldwide and is connected to the high morbidity and mortality rates [5]. Diabetes mellitus occurs in two main types: type 1 , and type 2 although there is a third diabetes type known as gestation diabetes though not universal [5].

The diagnostic threshold for the fasting plasma glucose for diabetes has been reduced to $7.0 \mathrm{mmol} / \mathrm{l}(126 \mathrm{mg} / \mathrm{dl})$, while studies have introduced the impaired fasting glucose (fasting plasma glucose $6.1-6.9 \mathrm{mmol} / \mathrm{l}[110-125 \mathrm{mg} / \mathrm{dl}]$ ) as a 
new type of intermediary glucose metabolism. These changes reveal that the ancient fasting minimum did not compare the 2-h (post load) threshold well and that both the micro-29 and the macrovascular disease manifest at comparatively reduced levels of the fasting glucose than it was previously thought. Besides, Impaired Glucose Tolerance (IGT) was a separate class in the past but is now classified as a specific level in the natural history of abnormal metabolism of carbohydrate. Furthermore, IGT is braced with impaired fasting glucose (6.1 $7.0 \mathrm{mmol} / \mathrm{l}$ [110 - $126 \mathrm{mg} / \mathrm{dl}]$ ) [6].

\section{2) Sub-Theme 2: Type 2 diabetes}

Type 2 diabetes is a long-term disease which causes an impact in the way bodies to handle glucose and sugar present in the blood. Many individuals with type 2 diabetes might be faced with the condition of pre-diabetes [7]. Pre-diabetes is a condition associated with an abnormality in the glucose level, though not high enough to be referred to as diabetes. Individuals with type 2 diabetes usually secrete insulin, but their cells have limited use or do not use it as required. This is traditionally known as insulin confrontation [7].

Type 2 diabetes is related to inadequate insulin or the insulin produced does not function properly. Most people are identified with type 2 diabetes and are common to the aged above 40 as well as overweight individuals. Mainly controlled by following a low sugar balanced diet, weight loss and perspective exercises [8].

At first, the pancreas secrets more insulin to try to get glucose into the cells. However, eventually it cannot keep up, and the sugar builds up in once blood instead. Some of the factors which lead to type 2 diabetes include the following [9]. Genes- the gene formation in DNA can affect the way one's body secretes insulin, excess weight overweight can cause insulin resistance [9].

Type two diabetes affects mostly kids and those who are obese. Metabolic Syndrome refers to patients with insulin resistance, who suffers from a condition of high blood glucose, more fat around waist line, high blood pressure, triglycerides and high amounts of cholesterol, a lot of Glucose from the liver in the event of low blood sugar, liver secretes and sends glucose [7]. During eating the level of blood sugar rises steadily causing the liver to slow down thereby storing glucose. In diabetic people, the liver does not stop and keep on producing more sugar [7].

Insulin resistance is a significant contributor to the pathophysiological pattern of the development of type 2 diabetes. Evidence of this provided by the longitudinal and cross-sectional inquisitions showing that insulin resistance occurs 10 20 years before the disease manifestation and that it best predicts whether or not a person will have diabetes at one time. Furthermore, insulin resistance affects the progressive dysfunction of beta cells of type 2 diabetes by placing more requirements on the $\beta$-cell to perpetuate hypersecretion of insulin. The exact insulin resistance mechanism(s) that render the $\beta$-cells dysfunctional remain(s) unknown, but some researchers hypothesize that the root of insulin resistance is 
also directly to blame for the $\beta$-cell abnormality (i.e., lipotoxicity), [1].

\section{3) Sub-Theme 3: Diabetes Mellitus complication.}

The complications of Diabetes Mellitus are categorized into macrovascular and microvascular. Microvascular complications consist of Nephropathy, Retinopathy, and Neuropathy, [9]. On the other hand, Macrovascular complications comprise peripheral arterial diseases, stroke, and coronary artery disease. Diabetes leads to acute complications associated with the rise of hyperglycemic crises, termed as one of the long-term progressive complications of diabetes and has been linked to devastating and life-threatening across many diabetic patients [9].

Foot complication among many patients who have Type 2 diabetes has caused high mortality rate and morbidity. Consequently, the pandemic has attracted high cost and health care utilization. Among the diabetic population, patients who have peripheral neuropathy, as well as peripheral arterial disease (PAD), are at the high exposure of foot ulceration as well as infection and other infection leading to a reduced amputation. Over the past years, the rates of amputation have gone low; they remain high as compare to persons who are non-diabetic [10].

\section{4) Sub-Theme 4: Diabetic Foot.}

Diabetic foot is a deep sore which is on foot. It can also appear in a shallow red crater which is on the upper surface skin. Diabetic patients, as well as those with a less developed circulation, are at high chances of developing foot ulcers. Foot ulcers are as a result of diabetic neuropathy or any other vascular disease [11]. Diabetic people who have any common foot problem can lead to other serious infections, and therefore foot care is critical. It is not easy to heal a foot ulcer diabetic people are very allergic to even a small ulcer. If an infection is manifested and it is not treated immediately, it can develop to any of the following: gangrene, it is an area of dead tissue which is as a result of little blood flow. An abscess, cellulitis, an infection which spreads to the skin and the underlying fat, a bone infection known as osteomyelitis. Most of the severe infections among diabetic people on some parts such as the toe, foot or lower are a start of foot ulcers [12].

\section{5) Sub-Theme 5: Early detection of diabetic foot ulceration.}

Before highlighting the early signs of diabetic foot ulcerations, it is crucial to mention some of the pathophysiological changes that usually arise from the functionality changes occurring due to injuries. The two main pathophysiological changes occurring in diabetes are atherosclerosis and peripheral neuropathy [13]. Essentially, diabetic foot ulceration usually happens when there is an injury to nerve damage, a condition referred to as neuropathy. Furthermore, individuals diagnosed with diabetes are more predisposed to develop atherosclerosis whereby the arteriolar hyalinosis thicken. Consequently, this reduces the amount of blood circulation to the parts of the body that are injured there by disturbing the body's healing process [13]. 
Treatment of diabetic foot is complicated and debilitating to treat. Tissue debridement is considered as part of standard care; debridement is regarded as the most effective treatment option for diabetic foot care. Debridement allows for the removal of callus, necrotic tissue, as well as the elimination of bacterial biofilms [14]. Depending on the severity of the diabetic foot ulceration, which can also be assessed through concept mapping, there are different types of debridement techniques [14].

As a management technique, the hydrogel dressing was the most suitable management technique for patients suffering from diabetes foot ulcer. Also more effective in improving the healing process of superficial diabetic foot ulcers as compared to foams which were found to be effective in healing deep foot ulcers. Hence, providers may classify this information during concept mapping and as such, educate the patient on the most effective dressing approach that can be used depending on the depth of the foot ulcerations [1].

Other than hydrogel dressing, there were also different types of dressing used for patients with diabetic foot ulcers. One, hydrocolloid dressings used for wounds that have light draining's [1]. This is the second most used type of dressing after hydrogel. They are absorbent and can be left on the wound for several days. Second, alginate dressing that is used for injuries that have a lot of drainage and those that are in the severe and advanced stages. They are absorbent and also bacteriostatic and usually need to be wet before removing them from the wound. Third, collagen dressing used for ulcers that have spread and cover a large area of the foot [1].

Prevention is a core component in reducing the prevalence rate of diabetes foot ulceration among patients and as such, it is considered an integral part of diabetes foot care practice. [15] affirmed that it is paramount for not only health care providers but also diabetic patients to have the requisite knowledge on some of the pathophysiology and the predisposing risk factors of diabetic foot ulcerations. Therefore self-regulation is imperative.

6) Sub-Theme 6: Self -efficacy and Behavior.

Self-efficacy also defined as confidence is the positive self-belief in all chances to complete a given task and produce favorable outcomes. Self-efficacy is a virtual worth of having as it plays vital roles in our lives [16]. Behaviors can be defined set of how a person acts or speaks. It is a repetition of individual acts of talking as well as engaging in any activity [16].

Self-efficacy and behaviors are significant factors in self-care for people who have diabetes condition. Self-efficacy and diabetes management are directly related in that one has to set focus in glycemic control. Diabetes is one of the prevalent diseases in the world. There are different uncontrollable factors which need to be monitored in self-caring of diabetes for managing and preventing early and late effects of the disorders [17].

\subsubsection{Theme 2 Concept Mapping in Health Education}

1) Sub-Theme 1: Health education tool 
A concept map is useful as a tool for presenting evidence-based treatment and management strategies for patients that have developed foot problem [18].

Concept maps were derived from David Ausubel's theory, the critical learning theory which states that knowledge should be significantly relevant, understood, and well-integrated rather than developed arbitrarily and based on exclusive memorization [18].

For instance, some of the early clinical signs and symptoms that are always contained in concepts that can be used in the early detection of the disease include the increase of pain in a localized area of the extremities. The presence of erythema or rather the abnormal darkening of the ethnic skin, the presence of edema, the presence of purulent and sanguineous exudates can also be an early detection sign [19]. Subsequently, health care providers and nurses may use these clinical symptoms contained in concept maps to evaluate the subjective experience of pain depending on the response offered by the patient. For instance, if the patient responds that he has developed pain in recent months, this may be indicative of the early onset of foot ulcerations [19].

The use of concept mapping is considered the most effective strategy in highlighting both the pathophysiology and the risk factors of diabetes foot ulceration. Concept mapping allows both providers and patients to capture some of the contributing factors of diabetes foot ulcerations such as deformity, minor trauma, peripheral artery diseases, and deformity [20]. Other additional factors that contribute to the development of diabetic foot ulceration include factors such as age advancement as well as comorbidities such as heart failure and end-stage renal disease. Subsequently, providers and patients can be able to adequately gauge whether or not they are at risk of developing the condition [20].

2) Sub-Theme 2 concept map prevention and management of diabetic foot car

Concept maps are also useful in highlighting some of the preventive strategies that diabetic patients can utilize to cushion themselves against the risk of developing diabetic foot ulcerations. [21] asserts that broad concepts map highlight preventive measures that enlighten patients such as nail and skin care, blood pressure control, and lifestyle modification. For instance, in nail and skin care, concepts maps offer standard guidelines that inculcate patients the different ways that they can be able to maintain the integrity of their skin [18].

Foot care is a significant activity more so to diabetes patients. Problems emanating from foot are a common complication involved in this condition. Feet can be affected in some ways, when blood supply is not enough, leading to poor healing. Nerve damage in the feet can also lead to a loss of feeling. When a diabetic patient suffers from nerve loss will be unable to realize blisters or cuts, which may develop later to ulcers [22]. The plague of food problems can, however, be avoided by taking care of one's feet and taking immediate action if a problem is noted. Consequently, feet can be checked at least once annually by a 
podiatrist to detect feet problems early in advance before the problem gets out of hand [22].

In an attempt to reduce the number of people affected by diabetic foot, it is essential to implement acts that revolve around self-efficacy. Such actions are cost-effective as they do not require patients to make frequent visits to the physician. Diabetic foot care is enhanced through the use of concept maps. Concept maps have previously been used in conducting clinical education. Traditional education models focus on the disease instead of patient healthcare [15]. However, concept mapping considers patient healthcare as the primary aspect since it acknowledges that knowledge shaped by society and grows in a spiral [15].

The nursing rule calls for critical thinking according to [23] the importance of critical thinking has increased massively over the last ten decades while nursing educators have been emphasized to incorporate teaching skills which call for critical thinking among nursing students. [24], reported that the using of concept plotting in clinical learning of events and activities had been attributed to the improvement of critical thinking and also in motivating students to observe a patient, organize and prepare complex information expansively. Also, concept mapping enables nursing students in making a consent decision before partaking an empirical evaluation [24].

Concept map is used as a teaching strategy, where nursing schools use this method often for nursing students to learn more effectively and to be able to apply knowledge when working with diabetic patients. The analysis and examination required when treating a patient is the primary reason why nurses use the concept map because it helps them organize their ideas and information effectively and relate it with the different patients with different levels of diabetic foot ulcer. A concept map is primarily a tool for educating nurses on how to work in teams, how to analyze symptoms and how to treat patients accordingly.

\subsection{Gap in the Literature}

While searching electronic databases, the researcher was unable to find any published research effect of concept map as an educational intervention on diabetic foot among diabetic patients in KSA. Moreover only few studies in international level highlighted the application of concept map on diabetic patients. Consequently, this study will add information that fills existing research gaps, national and international, and will contribute to the development of competent evidence on the use of concept map as a diabetic foot care educational intervention for type 2 diabetic patients. Nursing intervention plays a cyclic part in clinical practice. The value of introducing this new method relies on encouraging patients with diabetes to participate in concept mapping as a method of foot care, and also will encourage health care provider to apply the concept map in any other type of patient's care and utilize it towards achieving the landmark goal of foot care. 


\subsection{Conclusion}

This review confirmed that there are a growing confirming literature about the application of concept maps as an effective nursing strategy that can be used by nurses and other health care professionals, in maintaing healthy feet and preventing further complications such as bruises, cuts and sores. Through concept mapping, there is a creation of awareness that can be used to promote effective care practices; the concept mapping that achieves in health education is an important part of the management of diabetic foot care. This education tool ensures that nurses are well prepared and equipped when working with patients with diabetes type 2 .

\section{Acknowledgements}

Special thanks to my supervisor and family members in particular my parents, for their endless support and encouragement.

\section{Authors Contribution}

Al Johani, R: Wrote the review and prepared the manuscript.

Al-Nagshabandi, E: Reviewed the manuscript.

\section{Conflicts of Interest}

The authors declare that they have no competing interests.

\section{References}

[1] Senthil, B., Shanmugam, S., Elangovan, S., Elumalai Chandramouli, P., Bhaskaran, S. and Ramesh, C. (2017) Comparative Study: Oral Mucosal Lesions, Signs and Symptoms in Diabetes Mellitus Patients with End Stage Renal Disease with Analogous Findings in Diabetes Mellitus Patients with Non-End Stage Renal Disease. Indian Journal of Dental Research, 28, 406. https://doi.org/10.4103/ijdr.IJDR 35016

[2] The World Health Organization Report on Diabetes 2016.

[3] Song, Y., Song, H.J., Han, H.R., Park, S.Y., Nam, S. and Kim, M.T. (2012) Unmet Needs for Social Support and Effects on Diabetes Self-Care Activities in Korean Americans with Type 2 Diabetes. Diabetes Educator, 38, 77-85. https://doi.org/10.1177/0145721711432456

[4] Kgasi, K. (2017) Diabetes Mellitus Type 1. Council for Medical Schemes Script. 1.

[5] Al-Rubeaan, K., Almashouq, M.K., Youssef, A.M., Al-Qumaidi, H., Al Derwish, M., Ouizi, S. and Masoodi, S.N. (2017) All-Cause Mortality among Diabetic Foot Patients and Related Risk Factors in Saudi Arabia. PLoS ONE, 12, e0188097. https://doi.org/10.1371/journal.pone.0188097

[6] Pender, N.J., Murdaugh, C.L. and Parsons, M.A. (2011) Health Promotion in Nursing Practice. 6th Edition, Prentice Hall, Upper Saddle River.

[7] Shaw, J.E., Sicree, R.A. and Zimmet, P.Z. (2010) Global Estimates of the Prevalence of Diabetes for 2010 and 2030. Diabetes Research and Clinical Practice, 87, 4-14. https://doi.org/10.1016/j.diabres.2009.10.007 
[8] Funnell, M.M., Brown, T.L., Childs, B.P., Haas, L.B., Hosey, G.M., Jensen, B. and Weiss, M.A. (2011) National Standards for Diabetes Self-Management Education. Diabetes Care, 34, S89-S96. https://doi.org/10.2337/dc11-S089

[9] Grant, T., Soriano, Y., Marantz, P.R., Nelson, I., Williams, E., Ramirez, D., et al. (2014) Community-Based Screening for Cardiovascular Disease and Diabetes Using HbA1c. American Journal of Preventive Medicine, 26, 271-275. https://doi.org/10.1016/j.amepre.2003.12.015

[10] Duke, S.A., Colagiuri, S. and Colagiuri, R. (2011) Individual Patient Education for People with Type 2 Diabetes Mellitus. Cochrane Database of Systematic Reviews, No. 1, CD005268.

[11] Sharma, S.K., Ghimire, A., Radhakrishnan, J., Thapa, L., Shrestha, N.R., Paudel, N. and Brodie, D. (2011) Prevalence of Hypertension, Obesity, Diabetes, and Metabolic Syndrome in Nepal. International Journal of Hypertension, 2011, Article ID: 821971. https://doi.org/10.4061/2011/821971

[12] Joshi, V.D., Malhotra, R., Lim, J.F., Østbye, T. and Wong, M. (2010) Validity and Reliability of the Expectations Regarding Aging (ERA-12) Instrument among Middle-Aged Singaporeans. Annals of the Academy of Medicine, Singapore, 39, 394-398.

[13] Hörnsten, Å., Stenlund, H., Lundman, B. and Sandström, H. (2010) Improvements in HbAlc Remain after 5 Years: A Follow up of an Educational Intervention Focusing on Patients' Understandings of Type 2 Diabetes. Diabetes Research and Clinical Practice, 81, 50-55. https://doi.org/10.1016/j.diabres.2008.02.005

[14] Pourhaji, F., Delshad, M.H., Ammari, A.A. and Pourhaji, R. (2016) Foot-Care Self-Efficacy Beliefs, Physical Self-Concept and Actual Foot-Care Behavior in People with Diabetes Mellitus. International Journal of Musculoskeletal Pain Prevention, 1, 101-107.

[15] Alexiadou, K. and Doupis, J. (2012) Management of Diabetic Foot Ulcers. Diabetes Therapy, 3, 4. https://doi.org/10.1007/s13300-012-0004-9

[16] King, H., Aubert, R.E. and Herman, W.H. (2013) Global Burden of Diabetes, 1995-2025: Prevalence, Numerical Estimates, and Projections. Diabetes Care, 21, 1414-1431. https://doi.org/10.2337/diacare.21.9.1414

[17] Piette, J.D., Resnicow, K., Choi, H. and Heisler, M. (2013) A Diabetes Peer Support Intervention That Improved Glycaemic Control: Mediators and Moderators of Intervention Effectiveness. Chronic Illness, 9, 258-267. https://doi.org/10.1177/1742395313476522

[18] Godet, M., Marchand, C., De Andrade, V. and Gagnayre, R. (2015) The Use of Concept Maps in the Therapeutic Patient Education Field: Preliminary Study with an Analysis of the Literature. Therapeutic Patient Education, 7, 20103-20112. https://doi.org/10.1051/tpe/2015014

[19] Formosa, C., Gatt, A. and Chockalingam, N. (2016) A Critical Evaluation of Existing Diabetic Foot Screening Guidelines. The Review of Diabetic Studies. RDS, 13, 158. https://doi.org/10.1900/RDS.2016.13.158

[20] Shouip, H.A. (2014) Diabetes Mellitus: Signs and Symptoms. Sinai University Press, North Sinai.

[21] Algshanen, M.A., Almuhanna, M.F., Almuhanna, A.M., Alghobaish, F.F., Alajji, N.A., Alabdullah, H.J., Almustafa, M.A., et al. (2017) Diabetic Foot Awareness among Diabetic Patients in Saudi Arabia. Egyptian Journal of Hospital Medicine, 68, 1289-1290. https://doi.org/10.12816/0039063 
[22] Rosland, A.M., Kieffer, E., Israel, B., Cofield, M., Palmisano, G., Sinco, B., Heisler, M., et al. (2012) When Is Social Support Important? The Association of Family Support and Professional Support with Specific Diabetes Self-Management Behaviours. Journal of General Internal Medicine, 23, 1992-1999.

https://doi.org/10.1007/s11606-008-0814-7

[23] Sinwar, P.D. (2015) The Diabetic Foot Management-Recent Advance. International Journal of Surgery, 15, 27-30. https://doi.org/10.1016/j.ijsu.2015.01.023

[24] Maneval, R.E., Filburn, M.J., Deringer, S.O. and Lum, G.D. (2015) Concept Mapping. Does It Improve Critical Thinking Ability in Practical Conflict of Interest. The Research Journal of the National League for Nursing, 32, 229-233.

https://doi.org/10.5480/1536-5026-32.4.229 